\title{
MULTIVARIABLE CONTROL USING THE SVD IN STEEL STRIP ROLLING
}

\author{
J.V. RINGWOOD \\ School of Electronic Engineering, Dublin City University, Glasnevin, Duhlin 9, Ireland
}

\begin{abstract}
Abstact. The shape control problem for a Sendzimir cold rolling ateal mill has been well documented (Grimble and Fotakis (1982), Ringwood and Grimble (1983), Ringwond and Grimble (1990), Ringwood et al. (1890)). However, application of the Singular Volue Decomposition (SVD) allows valuable insight to be gained into the control problem and produces a superior control strategy. In addition, singular values provide a natural basis for robustness anslysis which is important in the mill context, due to the multi-paes, multi-gchedule operation with resulting frequent changes in the plant parameters.
\end{abstract}

Key Words. Steel manufacture, rolling mills, multivariable systems, robuatnegs.

\section{SHAPE OF STEEL STRIP}

Shape (in the current context) refers to the stress distribution in steel strip. A strip with perfect shape has a uniform internal stress distribution, so that if cut into narrow strips, will lie flat on a flat surface. Bad shape can cause strip to buckle or tear (in the extreme). Shape measurement is performed by measuring a differential tension profile across the strip (see Fig.1) at 8 (modelled) equally-spaced points. The output of the system is therefore a profile, represented in vector form.

Strip shape may be controlled by bending the rolls of the mill, causing selective elongation of the strip at points where the rolls are closest. 'Long' or loose sections of the strip have associated compressive stress, while 'short' or tight sections suffer from tensile stress.

\section{SENDZIMIR MILL MODEL}

The Z-mill has an ASEA 'Stressometer' for measuing shape, located $5.91 \mathrm{~m}$ downstream of the rollgap. Two separate types of actuation for roll bending are provided (see Fig.1) - the 'As-U. Rolls' provide the equivalent of 8 independent oqually spaced point loads, while the First Intermediate Rolls are tapered, with lateral movement creating lective roll bending. Upper and lower sets of first intermediate rolls have opposite tapers, allowing both sides of the strip to be influenced equally, if necessary.

The $\mathrm{Z}$-mill, therefore, has 8 outputs and 10 inputs (8 AUR and $2 \mathrm{FIR}$ ). The rolling cluster is the most complex part of the system and accounts for all of the interaction between the 8 (modelled) paths in the system. Linearized gain matrices $\left(G_{\mathbb{Q}} \in \Re^{8 \times 8}\right.$ for the AUR's and $G_{j} \in \Re^{8 \times 2}$ for the
FIR's) relate changes in the roll-gap shape profile to changes in the positions of the AUR and FIR actuators respectively. Diagonal dynamical blocks account for the actuators, strip dynamics (between roll-gap and shapemeter) and the shapemeter.

Although the actuators are nonlinear (represented by Fig.2), the application of describing-function-based linearizing precompensators (Ringwood and Grimble (1990)) give an overall linearized model of:

$$
G(s)=g(s)\left[\begin{array}{ll}
G_{a} & G_{i}
\end{array}\right]
$$

where

$$
g(s)=\frac{e^{-0.582 s}}{(1+1.064 s)(1+0.74 s)(1+2 s)}
$$

for a medium $(5 \mathrm{~m} / \mathrm{s})$ strip spoed.

\section{FEEDBACK CONTROLLER DESIGN}

Classical approaches to the multivariable design problem suggest diagonalisation, and from equation (1) it would seem possible to decouple the system exactly at all frequencies. However, two factors complicate the issue. Firstly, the matrix:

$$
G_{m}=\left[\begin{array}{ll}
G_{a} & G_{i}
\end{array}\right] \in \Re^{8 \times 10}(3)
$$

is non-square and secondly, $G_{a}$ is not full rank, resulting in a rank number of less than 8 for $G_{m}$.

\subsection{Brevious Aporoachas}

In Grimble and Fotakis (1982), Ringwood and Grimble (1983) and Ringwood et al. (1990), 
attention is focussed on the AUR system oniy. The optimal control formulation of Ringwood and Grimble (1983) suggests precompensating the forward path with $G_{m}{ }^{-1}$, and the singularity problem is addressed in two ways. In Grimble and Fotakis (1982), the shape profile output is parameterised in terms of coefficients of 1 st to 4th order polynomial profiles present in the output. A $4 \times 8$ parameterisation matrix reduces the effective number of outputs to 4 , with a complementary $(8 \times 4)$ deparameterisation applied to the inputs. The resulting $4 \times 4$ system is now full rank and can be inverted.

In Ringwood et al. (1990), an effective pseudoinverse of $\mathrm{G}_{\mathrm{m}}$ is obtained by decomposing the system into its eigencomponents and neglecting the 'small' eigenvalues. This approach has much similarity with that described previously; but the effective parameterisation matrix is formed by the four largest eigenvectors, which represent the natural bending modes of the mill. The diagonalising precompensator for this case is itself diagonal.

In Ringwood and Grimble (1990), the full system is considered. Input and output parameterisations (as before) raduce the system to a full rank $4 \times 6$ system, with a Moore-Penrose right inverse (Ben-Israel and Greville (1974)) used to diagonalise the system. Such a choice of (non-unique) right inverse minimises the norm of the control input vector.

\subsection{SVD Approgach}

The SVD (Klema and Laub (1980)) is an appropriate design tool for the current problem for a number of reasons:

- The SVD has a diagonalising property for non-square systems

- The singular value spectrum is a good indicator of singularity (better than eigenspectrum)

- An SVD-based contraller provides a natural basis for robustness development

A typical singular value spectrum for $G_{m}$ is ovaluated as:

$$
\begin{array}{llllllll}
9.96 & 7.60 & 4.19 & 1.48 & 0.33 & 0.25 & 0.09 & 0.02
\end{array}
$$

Clearly, a separation condition exists such that:

$$
\left.\left.\mu_{1}=\min _{1 \leq i \leq 4}\left|\sigma_{i}\right|\right\rangle\right\rangle \max _{3 \leq i \leq 8}\left|\sigma_{i}\right|=\mu_{2}
$$

Therefore, it would seem appropriate to concentrate the control design on the modes corresponding to the larger singular values. There are three factors which support this decision:

- An inverse which relies on the full singular value spectrum is likely to be sensitive to small variations in $G_{m}$, due to its relatively poor condition number (ratio of min. to max. singular values). $G_{m}$ is known to contain modelling inaccuracies and two different modelling exercises (Gunawardene (1982), Dutton (1983)) have resulted in poor agreement on the values of the matrix gains.

- Under normal rolling conditions, no attempt is made to control shape components representing polynomial profiles greater than fourth order due to danger of fracturing the back-up rolls. Fig.3 shows the first four left singular vectors, indicating the low order natural bending modes present in the mill.

- The size of the smallest four singular values (effective gains associated with polynomial orders $4->$ ) indicate the large amount of control effort required to set up high order roll bending.

With reference to the separation condition in (4) above, the system is decomposed into a partitioned SVD as follows:

$$
G(s)=g(s)\left[\begin{array}{ll}
U_{1} & U_{2}
\end{array}\right]\left[\begin{array}{ccc}
\Sigma_{1} & 0 & 0 \\
0 & \Sigma_{2} & 0
\end{array}\right]\left[\begin{array}{l}
V_{1}^{T} \\
V_{2}^{T}
\end{array}\right]
$$

where:

$U_{1}, U_{2} \in \Re^{8 \times 4}, V_{1} \in \Re^{10 \times 4}, V_{2} \in \Re^{10 \times 6}, \Sigma_{1}, \Sigma_{2} \in \Re^{4 \times 4}$

Equation (5) can alternatively be expressed as:

$$
G(s)=g(s)\left\{U_{1} \Sigma_{1} V_{1}^{T}+U_{2} \Sigma_{2} V_{2}^{T}\right\}
$$

Now choose a forward path compensator:

$$
K(s)=k(s) K=k(s) V_{1} \Sigma_{1}^{-1} U_{1}^{T}
$$

so that the system is diagonalised with respect to the $\Sigma_{1}$ singular values and the high order shape profiles present in the output are jgnored, via the $\mathrm{U}_{1} \mathrm{~T}$ parameterisation. $\mathrm{k}(\mathrm{s})$ is a scalar transfer function chosen to give suitable closed-loop dynamics. Fig.4 shows the compensated system, where the reference and output are specified as (8-point) shape profiles. The dynamic system design was performed using classical (scalar) frequency response techniques to give:

$$
k(s)=\frac{200(2 s+1)^{2}}{(10(0 s+1)(0.9 s+1)}
$$

for a medium speed plant. A nonlinear simulation was used to assess the performance of the SVDbased controller. A uniformily flat shape profile was demanded (shape parameters $1 \rightarrow 4$ set $\approx 0$ ) with a constant, but nonzero, disturbance profile $(d(t))$ being introduced to simulate poor incoming strip shape (see Figs. 1 and 4 ). Fig.5 shows the shape profile variations with time. The initial shape profile (time $=0$ to 3 secs.) represents the shape disturbance appearing at the shapemeter. After 3 secs., control is applied and only a high (> $4^{\text {th }}$ ) order residual profile remains at the end of the simulation run. 


\section{A QUANTITATIVE ROBUSTNESS MEASURE}

The Z-mill processes more than 3500 different material sizes and types (with associated changes in roll diameters, taper gradients, etc). Since it is desirable to use one controller with a number of different mill schedules, it is important to have a measure of the allowable variations in $G_{m}$ which retain stability ( $g(s)$ is constant for a given strip speed). Singular values provide a good framework for, robustness analysis due to (i) their analogy with gain for scalar systems, and (ii) their relationship with matrix norms. In addition, since the Z-mill controller is SVD based, particularly simple robustness measures result. Robustness measures based on singular values are widely reported in the literature (e.g. Doyle (1979), Postlethwaite et al. (1981)). The analysis here follows follows the general spirit of these approaches.

Consider an additively perturbed Z-mill system, where a perturbation $\Delta_{m} \in \Re^{8 \times 10}$ has occurred in $\mathrm{G}_{\mathrm{m}}$ due to a pass or schedule change, with no corresponding change in the controller matrices. The stability of the perturbed system is described by the return difference as:

$$
\tilde{\sigma}\left[I_{\mathrm{g}}+\left(G_{m}+\Delta_{m}\right) K g k(s)\right]>0 \text { for } s \geq 0
$$

where the maximum and minimum singular values are denoted by $\bar{\sigma}[$.$] and \tilde{\sigma}[$.$] respectively.$ Since the square bracketed matrix in (9) is strictly proper and analytic and bounded in the interior of $D$, the suprema are achieved on the imaginary axis, so (9) may be replaced by:

$\tilde{\sigma}\left[I_{8}+\left(G_{m}+\Delta_{m}\right) K g k(j \omega)\right]>0$ for $\omega \geq 0$

Using the relation: $\quad \tilde{\sigma}(A+B) \geq \tilde{\sigma}(A)-\bar{\sigma}(B)$,

$\tilde{\sigma}\left[I_{g}+\left(G_{m} K g k(j \omega)\right]>\bar{\sigma}\left[\Delta_{m} K g k(j \omega)\right], \omega \geq 0\right.$

Using a second relation: $\bar{\sigma}(A B) \leq \bar{\sigma}(A) \bar{\sigma}(B)$,

$\tilde{\sigma}\left[I_{8}+\left(G_{m} K g k(j \omega)\right]>\bar{\sigma}\left(\Delta_{m}\right) \sigma \mid K g k(j \omega)\right], \omega 20(12)$

Equation (12) describes a condition for the stability of the perturbed system in terms of an upper bound on the max. singular value of the perturbation matrix. However, some further simplifications of eq. (12) are possible.

Some manipulation of the term $\vec{\sigma}[K g k(j \omega)]$ yields:

$$
\bar{\sigma}[K g k(j \omega)]=\frac{|g k(j \omega)|}{\sigma_{4}}
$$

It may also be shown that:

$$
\tilde{\sigma}\left(I+G_{m} K g k(j \omega)\right)=1
$$

The condition for the retention of stability may now be rewritten as:

$$
1>\bar{\sigma}\left(\Delta_{m}\right)\left(1 / \sigma_{4}\right) \sup _{\omega \geq 0}|g k(j \omega)|
$$

\section{PROPERTIES OF THE SVD CONTROLLER}

\subsection{Property 1}

The Z-mill shape control problem may be represented as a set of underdetermined equations - it is required to calculate 10 control inputs from 8 error signals (see Fig.4). The diagonalisation problem may be stated as: Determine $G^{+}$in (16) such that $y=e$, with respect to low (1st $\rightarrow 4$ th) order shape profiles:

$$
\begin{aligned}
& y=G_{m} G^{+} e \\
& u=G^{+} e
\end{aligned}
$$

where

$y$ and $e$ are the output and error vectors respectively and the dynamics are omitted for clarity. $\mathrm{G}^{+}$is the required right inverse matrix, and if evaluated using the singular value decomposition, is the Moore-Penrose inverse (Ben-Israel and Greville (1974)), which has the property of minimising the solution norm (in this case $u T_{u}$, the norm of the control vector). This is superior to the scheme of Ringwood and Grimble (1990) (R\&G scheme) which, although minimising the norm of a control vector $\left(\epsilon \Re^{6}\right)$, concentrates on the parameterised control vector, the control signals passing thorugh a further deparameterisation stage (to obtain 10 signals) before reaching the actuators.

\subsection{Property 2}

With regard to computational complexity of the controller, Both the SVD and R\&G schemes have $4 \times 8$ output parameterisations. Both schemes also have input deparameterisations, $8 \times 4$ for the $R \& G$ scheme and $10 \times 4$ for the SVD scheme. However, the compensating (diagonalising) matrix in the R\&G scheme is a full $6 \times 4$, whereas the SVD scheme has diagonal matrix of size $4\left(\Sigma_{1}{ }^{-1}\right)$. The dynamic compensation for both schemes is identical. The following table summarises the computational requirements:

\begin{tabular}{|l|l|l|}
\hline Operation & R\&G & SVD \\
\hline o/p Param. & $32(x) \& 28(+)$ & $32(x) \& 28(+)$ \\
\hline V/p Deparam. & $32(x) \& 24(+)$ & $40(x) \& 30(+)$ \\
\hline Compensat. & $24(x) \& 18(+)$ & $4(x)$ \\
\hline Total & $88(x) \& 70(+)$ & $76(x) \& 58(+)$ \\
\hline
\end{tabular}

The reason for the lower computational effort for the SVD scheme is that the parameterisation itself diagonalises the system, the compensator 
merely equalising the gains in each of the resultant 4 separate loops. It would seem that this scheme concentrates on the 'natural' bending modes in the mill, without forcing an alien paramerterisation as in the R\&G scheme.

\section{CONCLUSION}

The Z-mill shape control problem has been recast in an SVD framework. This would seem to be the natural setting for the problem, considering such features as control signal norm minimisation, ease of decoupling and basis for robustness calculations. The resulting controller is straightforward to implement, and robustness to variations in the mill matrices is guaranteed within specific bounds calculated from equation (15). These may be evaluated a priori, since the mill setup is known for each schedule and mill matrices can be evaluated from the static model (Gunawardene (1982)), giving concise guidelines regarding the number of controller matrices required to cover all operating conditions.

\section{REFERENCES}

Ben-Israel, A. and Greville, T.N.E. (1974) Generalised Inverses - Theory and Applications. Wiley Interscience.

Doyle, J.C. (1979) Robustness of multiloop linear foedback systems, Proc. 17 $17^{\text {th }}$ IEEE Conf. on Decision and Control, San Diego.

Dutton, K. (1983) An Investigation into the Design and Performance of a Shape Control System for a Sendzimir Mill, Ph.D Thesis, Sheffieid City Polytechnic.

Grimble, M.J. and Fotakis, J. (1982) The design of shape control systems for Sendzimir mills, IEEE Trans. Auto. Con., AC.27, pp 656-666.

Gunawardene, G.W.D.M. (1982) Static model development for the Sendzimir Cold Rolling Mill, PhD Thesis, Sheffield City Polytechnic.

Klema, V.C. and Laub, A.J. (1980) The singular value decomposition: its computation and some applications, IEEE Trans. Auto. Con., AC-25, No. 2.

Postlethwaite, I., Edmunds, J.M. and MacFarlane, A.G.J. (1981) Principal gains and principal phases in the analysis of linear multivariable feedback systems, IEEE Trans. Auto. Con., AC.26, No.1.

Ringwood, J.V. and Grimble, M.J. (1983) An optimal output feedback solution to the strip shape multivariable control problem, Proc. IASTED Symp. on Applied Control and Identification, Copenhagen.

Ringwood, J.V. and Grimble, M.J. (1990) Shape control in Sendzimir mills using both crown and intermediate roll actuators, IEEE Trans. Auto. Con., AC-35, No.4.

Ringwood, J.V., Owens, D.H. and Grimble, M.J. (1990) Feedback design of a canonical multivariable system with application to shape control in Sendzimir mills, Proc, American Conirol Conference (ACC 90), San Diego.

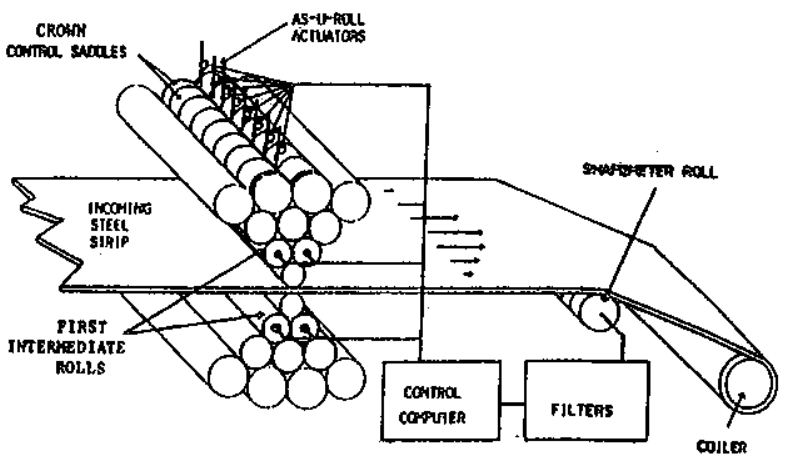

Figure 1 : Sendzimir 20-roll Cold Rolling Steel Mtll

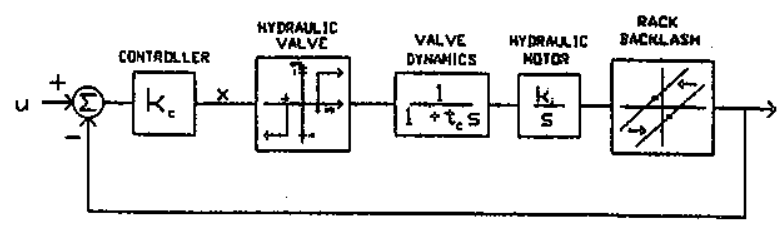

Fig. 2 : Actuator Block Diggram
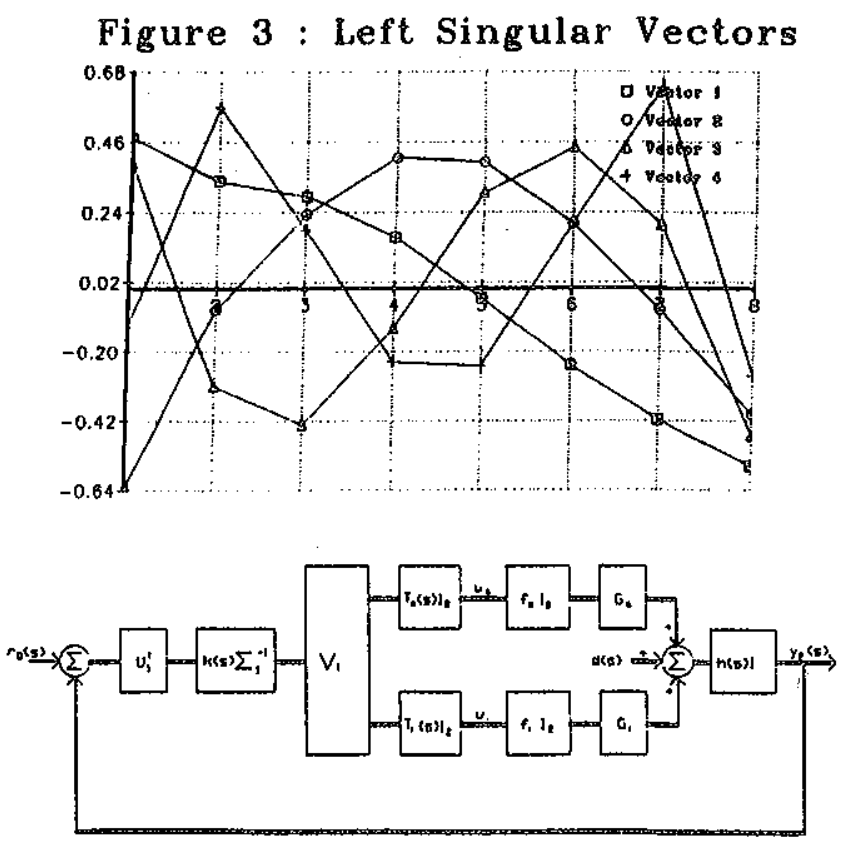

Fig. $4: 8 \times 8$ Compensated System

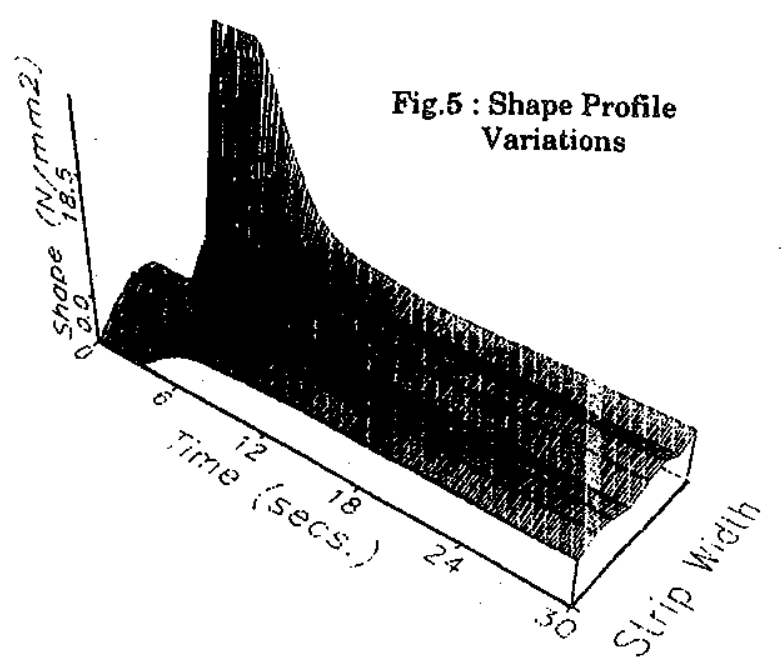

